TITLE:

\title{
Evaluation of 3D printed buckyball- shaped cathodes of titanium and stainless-steel for IEC fusion system
}

\section{$\operatorname{AUTHOR}(\mathrm{S})$ :}

Bakr, Mahmoud; Wulfkühler, Jan-Philipp; Mukai, Keisuke; Masuda, Kai; Tajmar, Martin; Konishi, Satoshi

\section{CITATION:}

Bakr, Mahmoud ...[et al]. Evaluation of 3D printed buckyball-shaped cathodes of titanium and stainless-steel for IEC fusion system. Physics of Plasmas 2021, 28(1): 012706.

\section{ISSUE DATE:}

2021

URL:

http://hdl.handle.net/2433/261719

\section{RIGHT:}

This article may be downloaded for personal use only. Any other use requires prior permission of the author and AIP Publishing. This article appeared in Physics of Plasmas 28, 012706 (2021) and may be found at https://doi.org/10.1063/5.0033342; The full-text file will be made open to the public on 21 January 2022 in accordance with publisher's 'Terms and Conditions for Self-Archiving'. 


\section{Evaluation of 3D printed buckyball-shaped cathodes of titanium and stainless-steel for IEC fusion system}

Cite as: Phys. Plasmas 28, 012706 (2021); https://doi.org/10.1063/5.0033342

Submitted: 15 October 2020 . Accepted: 31 December 2020 . Published Online: 21 January 2021

(D) Mahmoud Bakr, Jan-Philipp Wulfkühler, (D) Keisuke Mukai, (D) Kai Masuda, (D) Martin Tajmar, and Satoshi Konishi
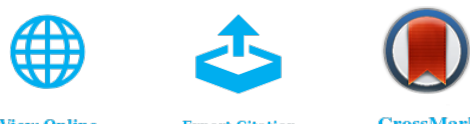

\section{ARTICLES YOU MAY BE INTERESTED IN}

First observation of increased DT yield over prediction due to addition of hydrogen

Physics of Plasmas 28, 012707 (2021); https://doi.org/10.1063/5.0030852

Characterization of fusion plasmas in the cylindrical DTU inertial electrostatic confinement device

Physics of Plasmas 27, 083515 (2020); https://doi.org/10.1063/5.0013013

Direct measurements of the 3D plasma velocity in single-helical-axis RFP plasmas

Physics of Plasmas 28, 012510 (2021); https://doi.org/10.1063/5.0025696 


\title{
Evaluation of 3D printed buckyball-shaped cathodes of titanium and stainless-steel for IEC fusion system
}

Cite as: Phys. Plasmas 28, 012706 (2021); doi: 10.1063/5.0033342

Submitted: 15 October 2020 - Accepted: 31 December 2020 .

Published Online: 21 January 2021

Mahmoud Bakr, ${ }^{1,2, a)}$ (D Jan-Philipp Wulfkühler, ${ }^{3}$ Keisuke Mukai, ${ }^{1}$ (D Kai Masuda, ${ }^{4}$ (D) Martin Tajmar, ${ }^{3}$ and Satoshi Konishi ${ }^{\top}$

\author{
AFFILIATIONS \\ ${ }^{1}$ Institute of Advanced Energy, Kyoto University Kyoto, 611-011 Japan \\ ${ }^{2}$ Physics Department, Faculty of Science, Assiut University Assiut, 71516, Egypt \\ ${ }^{3}$ Institute of Aerospace Engineering, Technische Universität Dresden, 01062, Germany \\ ${ }^{4}$ Rokkasho Fusion Institute, National Institutes for Quantum and Radiological Science and Technology, 039-3212, Japan \\ a) Author to whom correspondence should be addressed: bakr@iae.kyoto-u.ac.jp
}

\begin{abstract}
An inertial electrostatic confinement (IEC) fusion device accelerates ions, such as deuterium (D) or tritium (T), to produce nuclear fusion and generate neutrons. The IEC's straightforward configuration consists of a concentric spherical transparent cathode at a negative bias surrounded by a grounded spherical anode. The effects of cathode properties on the neutron production rate (NPR) remain, to date, inadequately studied. This study aims to determine the impact of the cathode material on the NPR by investigating fusion reactions on the cathode surface. Two buckyball-shaped cathodes made of stainless steel (SS) and titanium (Ti), both of $5 \mathrm{~cm}$ diameter, fabricated by selective laser melting and 3D printing, are used for this investigation. A SS spherical chamber of $25 \mathrm{~cm}$ inner diameter is used as an anode in this experiment. A performance evaluation of surface fusion reaction in the IEC using SS and Ti grids is conducted by examining the NPR as a function of the applied voltage and grid currents at different gas pressures. So far, IEC with Ti and SS cathodes achieves NPRs of 2.32 and $1.41 \times 10^{7} \mathrm{n} / \mathrm{s}$, respectively, at $5.6 \mathrm{~kW}(70 \mathrm{kV}, 80 \mathrm{~mA})$. The normalized NPRs (NPR/I-cathode) from IEC using SS and Ti cathodes are compared. The results demonstrate that fusion reaction occurs on the cathode surface, and fusion increases with the applied voltage. The measured NPR/I-cathode using the Ti cathode is higher than that of the SS cathode by factors of $1.36-1.64$ across the $20-70 \mathrm{kV}$ range. Moreover, fusion on the Ti cathode surface enhances the total NPR significantly compared to the SS cathode under the same conditions. The Ti's considerable ability to accumulate D ions and molecules compared with that of SS explains the difference of measured NPR results.
\end{abstract}

Published under license by AIP Publishing. https://doi.org/10.1063/5.0033342

\section{INTRODUCTION}

Inertial electrostatic confinement (IEC) is used to accelerate ions, such as deuterium (D) or tritium (T), to produce fusion reactions and generate neutrons using an electric potential between two electrodes. The IEC concept goes back to Elmore in 1959, ${ }^{1}$ after which Farnsworth patented its design; ${ }^{2}$ shortly afterward, additional work on IEC was done by Hirsch. ${ }^{3}$ IEC has been studied intensively during the last three decades, and its current, compact design provides a smallscale neutron source for diverse applications worldwide. ${ }^{4-13}$ The ideal configuration of an IEC device is a spherical grid cathode surrounded by a spherical anode. A potential difference of tens of $\mathrm{kV}$ is applied between the electrodes to establish a potential well for trapping ions and beginning discharge. Consequently, fusion reactions among ions take place, and products are, then, generated. An IEC device can produce neutrons, protons, and $\mathrm{x}$-rays through burning fuel, such as DD, $\mathrm{DT}$, and $\mathrm{D}^{3} \mathrm{He}$, in steady- or pulsed-state mode. IEC offers several advantages over other neutron sources, including a long-operation lifetime, without deterioration in strength and radioactivity, on-off capability, being safe and easy to handle, and compactness of configuration. Therefore, several applications have been developed by utilizing the IEC as a neutron source, such as nuclear security, medical, radiography, and neutron-activation analysis. ${ }^{14-16}$

Different types of collisions occur inside the IEC device to produce a fusion reaction. With the fact that the total fusion rate depends 
on a specific mixture of collisional reactions in the IEC device, these reactions are named "beam-beam fusion with a small donation," "beam-background gas fusion with a dominant contribution at low gas pressure," and "beam-surface fusion reactions with a significant contribution." The first reaction occurs between energetic ions. The second reactions occur between energetic ions and the background gas, while the third fusion reactions occur between the energetic ions and target atoms on the electrode surfaces. ${ }^{6,11,17}$ Details of the fusion reactions are given in Sec. III. Improving the neutron production rate (NPR) is of great importance in widening IEC applications' potential. Studies revealed that the NPR strongly depends on the input power, applied voltage, and current on the cathode on the IEC device. ${ }^{18,19}$ Some studies reported that the electrode shape and dimension influence neutron yield. ${ }^{6,20,21}$ Other studies focused on the anode material in the context of improving the NPR. ${ }^{11,22}$ Elsewhere studies use different cathode materials, operated in a cylindrical anode at $<0.1 \mathrm{~Pa}$ gas pressure and $<40 \mathrm{kV}$ applied voltage, in the context of cathode surface fusion and its significant contribution to the NPR. ${ }^{17}$ However, a high NPR from the IEC device is the keyword for widening the device reliability as a neutron generator for a user facility. Thus, a high operation regime, such as several $\mathrm{kW}$ input power and several Pa gas pressure, is mandatory to satisfy NPR users' demand. From this perspective, the influence of the grid material and fusion-reaction type inside the IEC on neutron yield is inadequately studied.

The present work examines the impact of the grid material on the NPR in a spherical IEC device by investigating the surface fusion reactions. Two grids comprising SS-316L (SS, stainless steel) and Ti$6 \mathrm{Al}-4 \mathrm{~V}$ (Ti, titanium), fabricated using selective laser melting and 3D printing in a buckyball-shape, ${ }^{23}$ are used for this investigation. The grids in this study are interesting for practical reasons, such as a large surface area and high symmetric geometry compared with those of conventional disk grids enabling the fusion reactions on the cathode surface, which is a motivation behind using this type of grids in this study. In Sec. II, the experimental setup and conditions are described. In Sec. III, the results and discussion are presented, followed by a summary in Sec. IV.

\section{EXPERIMENTAL SETUP AND CONDITIONS}

The IEC used in this experiment [Fig. 1(a)] consists of an anode made of SS with an inner diameter of $25 \mathrm{~cm}$, which works as a vacuum vessel, surrounded by a $5 \mathrm{~cm}$ water-cooling jacket. A set consisting of a rotary pump (301/min) and a turbo molecular pump (501/min) exhausts the chamber and maintains a base pressure of $\sim 10^{-5} \mathrm{~Pa}$. The experiments in this study use $\mathrm{D}_{2}$ gas. The standard operating gas pressure during discharge is $1-3 \mathrm{~Pa}$. The gas is introduced to the chamber from an external cylinder through a leaking valve controlled using a mass flow controller. Two buckyball-shaped grids, with the same geometry, fabricated from SS [Fig. 1(b)] and Ti, are investigated. The inner grid diameter is $5 \mathrm{~cm}$ with 32 spherical apertures; the diameter of each is $1.5 \mathrm{~cm}$, and the grid thickness is $0.15 \mathrm{~cm}$. The cathodes' transparency is calculated to be $74 \%$. A molybdenum (Moly) rod, $2.5 \mathrm{~cm}$ in length, is used to hold the cathode in the chamber's center and connect it to the feedthrough stick. A Glassman high-voltage power supply with a maximum power of $8 \mathrm{~kW}(100 \mathrm{kV}$ voltage and $80 \mathrm{~mA}$ current) is used throughout the experiment.

A helium-3 $\left({ }^{3} \mathrm{He}\right)$ detector counts neutrons through the reaction $\mathrm{D}(\mathrm{d}, \mathrm{n})^{3} \mathrm{He}$. The count signal is collected using the ORTIC package

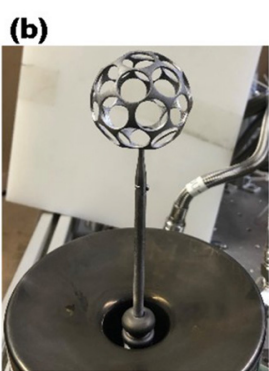

(C)

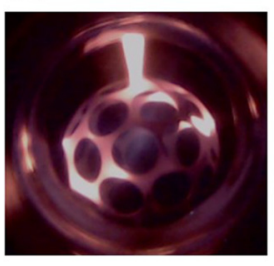

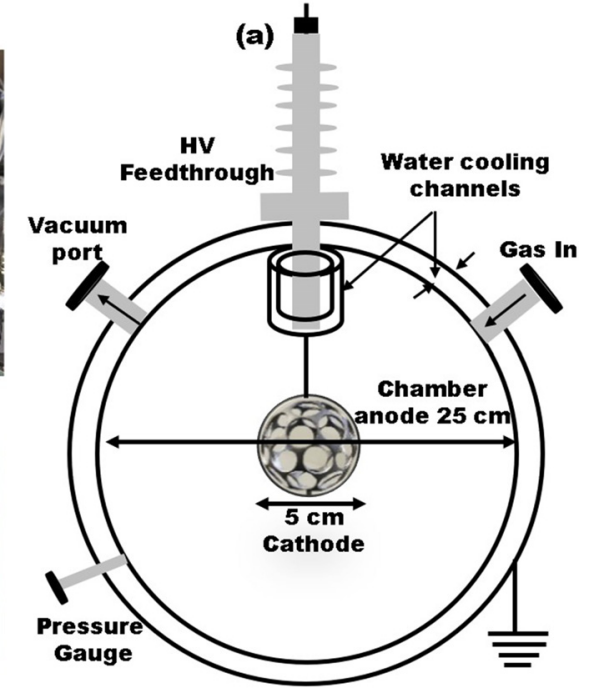

(a)

FIG. 1. (a) IEC chamber used in the experiment. (b) SS buckyball-shaped grid mounted on the feedthrough connection rod and high voltage stick. (c) SS cathode during discharge.

and then monitored using multichannel analyzer interfacing software. The neutron count system is calibrated using a ${ }^{252} \mathrm{Cf}$ source for $24 \mathrm{~h}$ before starting the experiment. The anode's inner surface temperature is kept constant by circulating water from an external chiller through a water jacket surrounding the anode [Fig. 1(a)], and the temperature of the circulated water is kept at $10^{\circ} \mathrm{C}$. During discharge, the cathode temperature is measured using an infrared thermometer (IR-FA from Chino), adjusted from one of the chamber viewports. The input and output parameters, such as applied voltage, grid current, gas flow, gas pressure, and water temperature of the anode surface, are controlled and monitored using Labview interfacing software and analog-to-digital converters. The input and output parameters are measured with a small fluctuation of \pm 0.01 , while the cathode temperature fluctuation range was $\pm 4{ }^{\circ} \mathrm{C}$. Prior to operation, the electrodes are conditioned, without supplying $\mathrm{D}_{2}$ gas to the IEC device, to remove absorbed gas impurities by increasing the cathode voltage from 1 to $100 \mathrm{kV}$, with a $0.1 \mathrm{~mA}$ grid current.

To begin discharge and thus produce neutrons in the IEC device, the chamber is filled with $\mathrm{D}_{2}$ gas. Currents of several tens of $\mathrm{mA}$ at tens of $\mathrm{kVs}$ are, then, applied to the cathode to generate electrons to begin the ionization and dissociation of $\mathrm{D}_{2}$ gas; then, the ions are accelerated to the center of the cathode. When plasma breakdown potential is achieved, discharge and fusion occur, generating neutrons. Before scanning the NPR, a discharge is conducted at $40 \mathrm{kV}$ and $50 \mathrm{~mA}$ for two hours to clean the cathodes' surfaces, as the grids are new in this experiment. DD fusion in the IEC device generates neutrons and protons, with energies of $2.45 \mathrm{MeV}$ and $3.02 \mathrm{MeV}$, respectively, as described in the following equation:

$$
D^{+}+D^{+} \rightarrow{ }^{3} \mathrm{He}+\mathrm{n}(2.45 \mathrm{MeV}) \text { or }{ }^{3} \mathrm{~T}+\mathrm{P}(3.02 \mathrm{MeV}) .
$$

The IEC system operates in constant-current mode, while the input current limits the system's discharge voltage. A fixed current is applied, and then, the gas pressure is tuned to achieve the system's discharge 
voltage. After the monitored voltage and gas pressure become stabilized, which takes an average of two minutes, the cathode temperature and NPR are measured. The NPRs are examined as a function of grid current-10 to $80 \mathrm{~mA}$ - at different applied voltages -20 to $70 \mathrm{kV}-$ for the IEC with Ti and SS cathodes. Figure 1(c) shows a photograph of the SS cathode during discharge.

\section{RESULTS AND DISCUSSION}

Pressure-voltage (P-V) curves, for a grid current of $10 \mathrm{~mA}$ and a voltage of $20-80 \mathrm{kV}$, using SS and Ti grids in the IEC, were measured to determine the breakdown voltages; the results are shown in Fig. 2. The breakdown voltage required to initiate IEC discharge depends on the gas pressure at a constant applied current. Figure 2 shows that the trend of the P-V curves obeys Paschen's law. Clearly, IEC breakdown in the Ti cathode occurs at lower pressure than in the SS cathode. This can be attributed to the Ti grid having a smaller work function than the SS grid, leading to increased thermionic emission of electrons and hence a higher ionization rate and lower breakdown voltage at a fixed pressure.

The grid current's influence on the NPR is examined from 10 to $80 \mathrm{~mA}$ at an applied voltage of $20-70 \mathrm{kV}$. The same measurements were performed four times, with a one-day interval, to study the effect of the cathodes' time trend on the NPR. The measured data collected from the fourth run are used to depict Figs. 3, 4, 5, and 7. Figure 3 shows the NPR as a function of grid current at applied voltages of 50 and $60 \mathrm{kV}$ for IEC with Ti and SS grids. The measured NPRs are plotted, with straight-line fitting. The measured NPR from the IEC with the Ti grid is higher than with the SS grid under the same conditions. A linear relationship between the NPRs and the cathode currents is observed from the fitting plots in Fig. 3. The slope and intercept values of the linear lines are different and depend on the applied voltage. The grid surfaces were new and then cleaned during the experiment. Nevertheless, enhancing the cathodes' performance is foreseen with the operation time. Improvement in the cathode performance with the operation time was observed of other cathodes in IEC systems, such as disk-type $^{11}$ or wire-type. $^{18}$

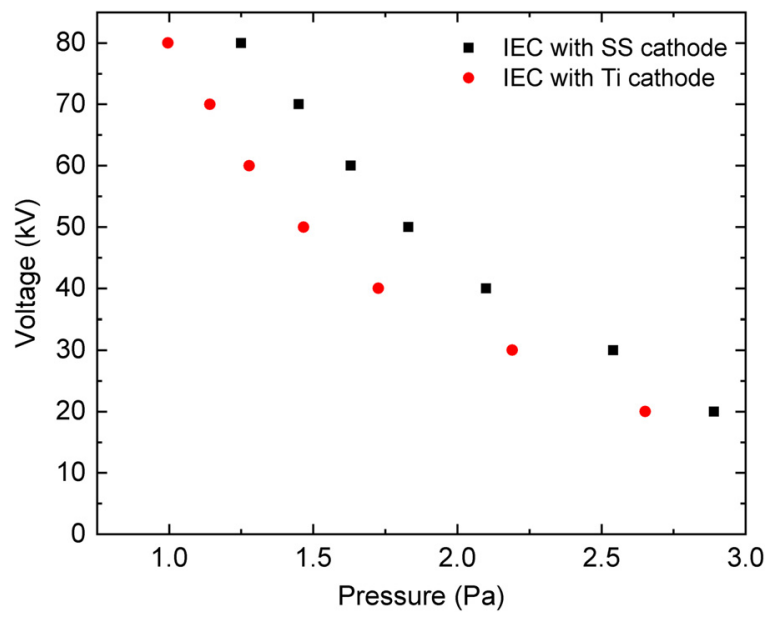

FIG. 2. Breakdown voltage vs gas pressure for IEC with Ti and SS grids for a grid current of $10 \mathrm{~mA}$.

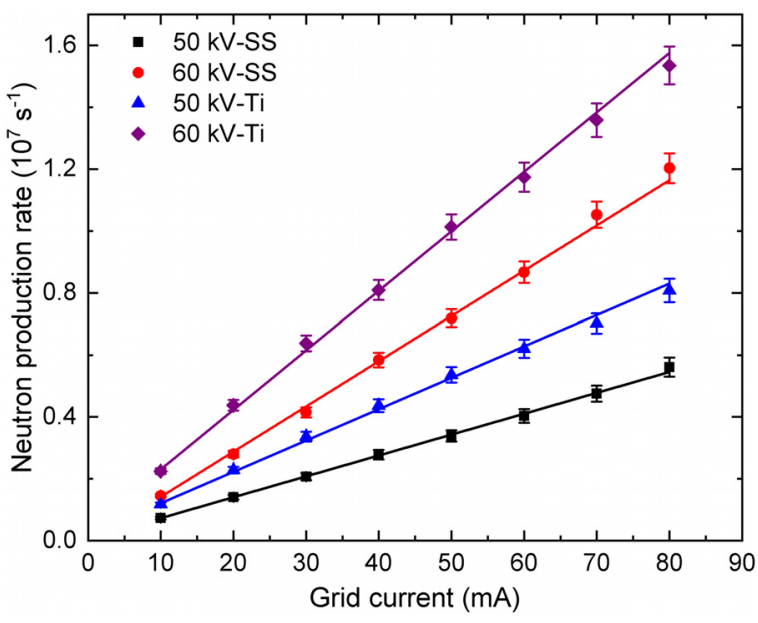

FIG. 3. NPR as a function of grid current for SS and Ti cathodes at applied voltages of 50 and $60 \mathrm{kV}$.

The relationship between the measured NPR and the grid current is analyzed. For an applied voltage of $20-70 \mathrm{kV}$, the relative increase in the NPR is compared in Fig. 4 with the grid current increase from 10 to $80 \mathrm{~mA}$ (for IEC with the SS cathode as an example). The data points represent the NPR relative increase with different current factors, ranging from $2(40 \mathrm{~mA} / 20 \mathrm{~mA})$ to $8(80 \mathrm{~mA} / 10 \mathrm{~mA})$ for different applied voltages. The NPR data points oscillate around the relative values; however, the average value is less than the relative values in general. For example, when the expected NPR relative increase is 3,5 , and 7 for IEC with the SS cathode, the average values are 2.92, 4.81, and 6.79 , respectively, while the corresponding NPR relative increases for the IEC with Ti are $2.94,4.85$, and 6.74 , respectively. The grid's performance could explain the difference between measured NPR relative increases and expected values. As the experiment's time frame is short, $\sim 30 \mathrm{~h}$ for each cathode, the grids' full capacities are not yet achieved. The effect of the operation time of the grids is discussed below.

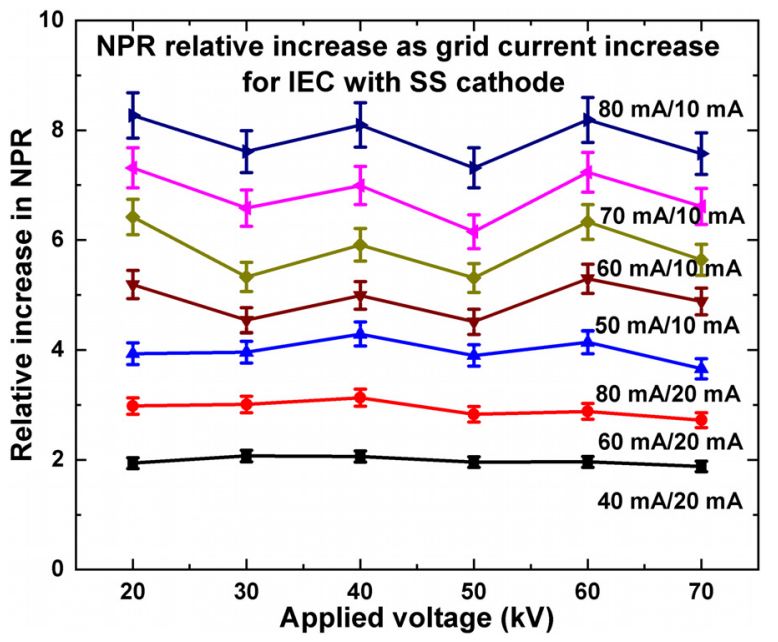

FIG. 4. The relative increase in the NPR as a function of applied voltage with the IEC grid currents' growth for IEC with the SS cathode. 


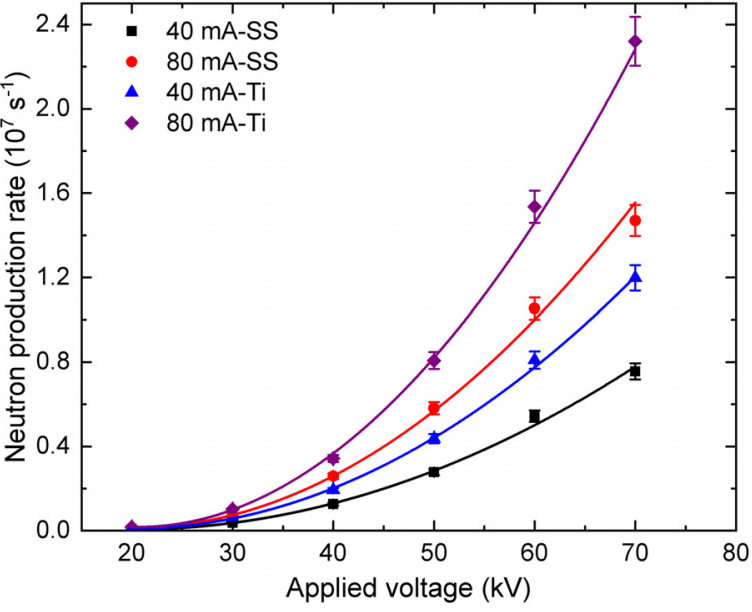

FIG. 5. NPR as a function of the applied voltage at 40 and $80 \mathrm{~mA}$ grid currents for the IEC with Ti and SS cathodes.

The influence of the applied voltage on the NPR for different grid currents is examined for the IEC with Ti and SS cathodes. The results are shown in Fig. 5 for 40 and $80 \mathrm{~mA}$ grid currents. The measured NPR results are first plotted, and then, the data are fitted using a second order polynomial. The voltage increase is expected to produce more than a linear increase in the NPR, equivalent to that of the DD fusion cross section with the D ions' kinetic energy. Apparently, the NPR of the Ti grid is superior to that of the SS grid for a given current and voltage. So far, achieved NPRs of the IEC device using Ti and SS cathodes at $5.6 \mathrm{~kW}(70 \mathrm{kV}$ and $80 \mathrm{~mA})$ are 2.32 and $1.41 \times 10^{7} \mathrm{n} / \mathrm{s}$, respectively.

We now investigate the NPR dependency on the cathode's material properties by monitoring cathode surface improvement with the number of runs. The NPR is recorded as a function of grid current from 10 to $80 \mathrm{~mA}$, at an applied voltage of $20-70 \mathrm{kV}$, for four runs in a row with a one-day interval between runs; the runs take place after cathode cleaning for the first time. The measured NPRs from the first and fourth runs at $70 \mathrm{kV}$ are shown in Fig. 6. The NPR from the IEC with the Ti cathode improves after four runs by a factor of 1.61-1.72, with 1.66 times on average. This improvement is interpreted as an enhancement in the cathode's surface performance after absorbing D and neutrals, which increases its probability of allowing surface fusion during operation. However, after four runs of using the SS cathode, the NPR increase is $1.05-1.17$, with 1.14 on average. This small NPR increase can be attributed to the removal of gas impurities from the cathode surface after heating above $800^{\circ} \mathrm{C}$ several times. It is noteworthy that the NPR improves 3.6 times from the same anode with a Moly disk cathode after ten years of intensive operation; ${ }^{11}$ this was explained by an enhancement in the anode and cathode performance via fusion on the anode surface. In the present study, the anode input is discarded due to the experiment's small time frame, and hence, the cathode is the primary source of the increase in the NPR. The notable improvement in Ti grid performance with the number of runs gives the merits of using Ti over SS.

Comparison of the normalized NPR (NPR/I-cathode) as a function of cathode voltage for IEC using Ti and SS grids for an

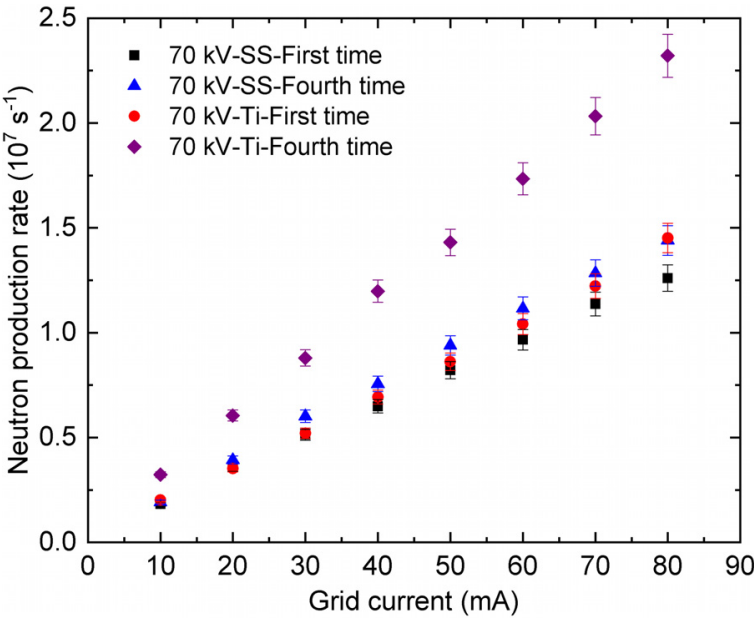

FIG. 6. Improvement in the NPR of the IEC with Ti and SS grids after four runs at $70 \mathrm{kV}$ applied voltage.

$80 \mathrm{~mA}$ current is shown in Fig. 7. The NPR/I-cathode from the IEC that uses the Ti grid is higher than the SS one for a given current and voltage. The results show that the NPR/I-cathode ratio between IEC with $\mathrm{Ti}$ and SS cathodes ranges from 1.36 to 1.64 in an applied voltage range from 20 to $70 \mathrm{kV}$. The NPR/I-cathode ratio changes as a function of voltage for all grid currents. To explain the NPR's reliance on the cathode materials shown in Figs. 6 and 7, one needs first to understand how the neutrons are generated and how different types of fusion reactions occur in the IEC device. The fusion reactions occurring in the IEC device and the factors affecting these reactions are thoroughly explained in the following subsections.

\section{A. Fusion rate in the IEC system}

Several atomic and molecular processes occur in the IEC space, such as ionization, dissociation, interchange, charge exchange, and

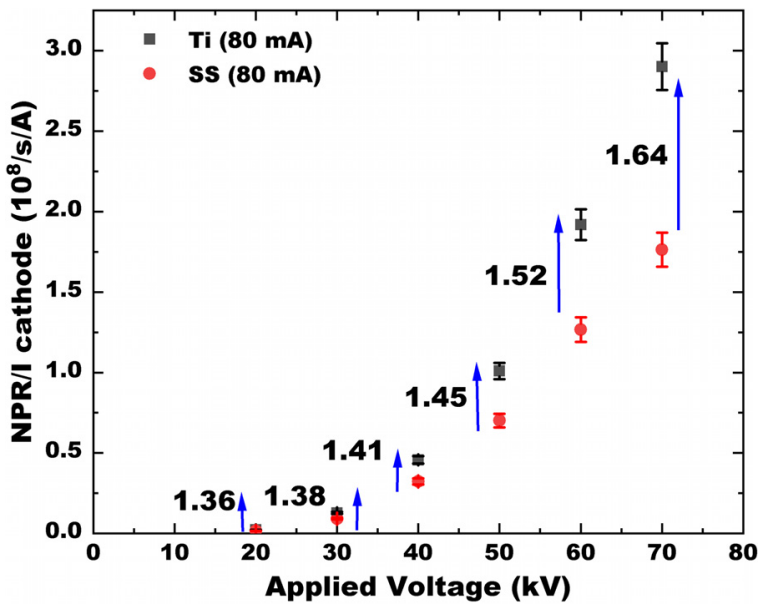

FIG. 7. NPR/I-cathode for the IEC system using Ti and SS grids at $80 \mathrm{~mA}$ current. 
recombination. These processes, summarized in Table I, control the fusion reactions in the IEC indirectly. Gaseous and surface fusion reactions are two types of reactions in which the system generates neutrons. Each type of reaction has a different cross section and probability; therefore, each makes a different contribution to the total NPR. The total NPR in the IEC device can be calculated approximately using the following equation:

$$
N P R \approx \frac{I \sigma}{e}\left(N_{\text {gas }}+N_{\text {surf }}\right),
$$

where $I$ is the cathode current, $e$ is the electron charge, and $\sigma$ is the distribution averaged fusion cross section of the fusion reaction, which depends on the applied voltage. $N_{\text {gas }}$ and $N_{\text {surf }}$ are the target gas density and the target surface density, respectively. $N_{g a s}$ represents the reaction between energetic ions and the background gas targets, while $N_{\text {surf }}$ includes fusion on the electrode's surface that generates neutrons. $N_{\text {surf }}$ can be separated into adsorbed and embedded target densities $N_{a d s}+N_{e m b}$ on the electrodes' surface. The beam-beam fusion, beam-background gas fusion, and beam-surface fusion reactions are described in detail below.

\section{Beam-beam fusion reaction}

This kind of reaction depends on the direct collisions between energetic ions inside the IEC center. The contribution of this fusion reaction to the total NPR, at low gas pressure $<2 \mathrm{~Pa}$ (nominal operation of the IEC system), is controlled by several factors: (i) ion density in the IEC space, which is generally low $\left(\sim 10^{9} \mathrm{~cm}^{-3}\right) ;{ }^{24}$ (ii) energy transfer from positive ions to background gas molecules through charge exchange and momentum transfer affects the efficiency of this type of fusion; ${ }^{25}$ and (iii) energy conversion between the applied voltage and ion energy is relatively small $(25 \%),{ }^{26}$ which reduces the merits of using the high fusion cross sections of DD at a high applied voltage. During the discharge, the IEC chamber comprises different deuterium species, such as $\mathrm{D}, \mathrm{D}_{2}, \mathrm{D}^{+}, \mathrm{D}^{+}$, and $\mathrm{D}^{+}{ }_{3}$, as seen in Table I, making the reaction processes complicated and challenging to be tracked. ${ }^{27}$ In contrast, the species mass analysis and Doppler shift are the only methods, so far, that can predict the predominant species in the IEC device. This topic may be discussed in a separate article, as it is beyond the current paper's scope. The cathodes were investigated under the same gas pressure conditions, grid current, and applied voltage. Therefore, the contribution of this fusion reaction type is constant and independent of the cathode material.

TABLE I. Atomic and molecular processes taking place in the IEC space during discharge.

\begin{tabular}{lc}
\hline \hline Ionization & $e+D_{2} \rightarrow D_{2}^{+}+2 e, e+D \rightarrow D^{+}+2 e$ \\
Dissociation & $e+D_{2} \rightarrow 2 D+e, e+D_{2}^{+} \rightarrow D^{+}+D+e$ \\
Ionization & $e+D_{2} \rightarrow D^{+}+D+2 e$ \\
+ Dissociation & \\
Charge exchange & $D^{+*}+D \rightarrow D^{*}+D^{+}, D_{2}^{+*}+D_{2} \rightarrow D_{2}^{*}+D_{2}^{+}$ \\
Interchange & $D_{2}+D_{2}^{+} \rightarrow D_{3}^{+}+D$ \\
$\begin{array}{l}\text { Dissociation } \\
+ \text { Recombination }\end{array}$ & $e+D_{3}^{+} \rightarrow D_{2}+D, e+D_{2}^{+} \rightarrow 2 D$ \\
\hline
\end{tabular}

\section{Beam-background gas fusion reaction}

This fusion reaction occurs between the energetic ions and the background gas, molecules, or neutrals generated from charge exchange, such as $\mathrm{D}, \mathrm{D}_{2}, \mathrm{D}^{+}$, and $\mathrm{D}^{+}$. The factors that control this type of fusion reaction are as follows: (i) the number of ions circulating inside the chamber before being lost to the electrodes, which depends on the gas pressure; (ii) the target gas density, which in turn depends on the gas pressure and cathode properties; and (iii) the number of circulations that an ion performs before being lost on the electrodes, which depends on the cathode transparency and its affinity to adsorb $\mathrm{D}$ atoms. Moreover, factors (ii) and (iii) in Sec. III A 1 also affect this fusion reaction type. The number of ions circulating depends on the cathode geometrical transparency $\eta$. The calculated transparency is $74 \%$ in the present study, assuming that the cathode radius is $2.5 \mathrm{~cm}$. The maximum number of circulations of the ions before being lost to electrodes is $\sim 1.6$ turns $\left[\delta=\eta /\left(1-\eta^{2}\right)\right]$, ${ }^{28}$ which is small compared with that of a conventional disk cathode type, with the same diameter and higher transparency.

The NPRs from IEC with the SS and Ti cathodes in the present study are comparable to those of an IEC with the same anode and Moly disk cathode with an $8 \mathrm{~cm}$ diameter and used intensively for two years. ${ }^{22}$ The parameters $\eta$ and $\delta$ for the disk grid, at $98 \%$ and 22 turns, respectively, are significantly high compared to those in the present study. Similar values of the measured NPRs from the buckyballshaped grid and disk cathode can be partially explained by the high geometrical symmetry of the buckyball-shaped grid compared with that of a disk grid. The buckyball-shaped grid's low transparency is compensated by high effective transparency. Disk grids usually feature differently shaped non-circular apertures, which buckyball-shaped grids in the present study do not, leading to asymmetric fields that prevent individual charged particles from remaining on the beam axis during the circulations. In addition, the high number of ion circulations in a disk grid enhances beam-background gas fusion reactions, which buckyball-shaped grids with low transparency such as $74 \%$ do not. On the other hand, the large surface area of buckyball-shaped grids enhances beam-surface target fusion compared with that of disk grids. The beam-surface fusion compensates the smaller beambackground gas fusion contribution in the total NPR from buckyballshaped grids and vice versa for the conventional disk cathode. The target gas density $N_{\text {gas }}$ of the present system can be calculated as follows: ${ }^{17}$

$$
N_{g a s}=2 \delta r P / k_{B} T,
$$

where $r$ is the cathode radius, $k_{B}$ is Boltzmann's constant, $P$ and $T$ are the gas pressure and temperature, respectively. $N_{\text {gas }}$ under the optimized operational conditions of the present IEC system is $\sim 1.35 \times 10^{15} \mathrm{~cm}^{-2}$, for $P=1 \mathrm{~Pa}$, and $T=20^{\circ} \mathrm{C}$. The radius, surface area, and operating conditions are the same for the investigated cathodes. Then, the transparency, number of ion circulations, and gas target density are equal for the present experiment. Therefore, the contribution of this type of reaction to the total NPR is almost constant and independent of the cathode material.

\section{Beam-surface fusion reaction}

This type of fusion reaction takes place at the surface of the electrodes (cathode and anode). The factors that control this type of fusion 
reaction are as follows: (i) the energy of ions (gained from the acceleration voltage) or neutrals generated by charge exchange and (ii) the ability of the electrode surface to absorb ions or neutrals (material properties). Factors (ii) and (iii) in Sec. III A 1 also influence the beamsurface fusion reaction. The ions and molecules attached to the electrode can be divided into adsorbed and embedded atoms based on their energy. In the present study, the anode is connected to a ground bias. Therefore, the anode is not reached by positive ions but is by neutrals. ${ }^{11}$ The water-cooling temperature of the anode surface and anode material is fixed while conducting the experiments on the Ti and SS cathodes. Thus, the contribution of the anode to the total fusion reaction is constant in the present study. However, the ions and neutrals collide with the cathode surface and initiate a fusion reaction with adsorbed or embedded ions and molecules. Therefore, only surface fusion reactions on the cathode are considered herein.

The capability of Ti and SS for accepting $\mathrm{H}$ and $\mathrm{D}^{+}$ions has been investigated experimentally in previous works. ${ }^{29-31}$ The embedded-ion surface density $N_{\text {emb }}$ at an ion energy of $25 \mathrm{keV}$ and a fluence of $1 \times 10^{18} \mathrm{~cm}^{-2}$ was estimated to be 6.0 and $2.3 \times 10^{18} \mathrm{~cm}^{-2}$ for Ti and SS surfaces, respectively. So, the $N_{e m b}$ of $\mathrm{D}^{+}$in the IEC device using the Ti grid is expected to be three times higher than that of the SS grid. Moreover, the Ti material is well known as a Getter material for adsorbing ions in vacuum systems. ${ }^{32}$ Thus, the $N_{a d s}$ value for the IEC with the Ti cathode is more significant than for IEC with the SS cathode. Hence, the IEC device's $N_{e m b}$ and $N_{a d s}$ values when using the Ti cathode are more considerable than when using the SS cathode. Consequently, more fusion reactions are expected at the $\mathrm{Ti}$ cathode surface compared with the SS cathode surface.

Simple diffusion calculations concerning $\mathrm{D}^{+}$ions, with energies of $0.01-100 \mathrm{keV}$ inside Ti and SS materials at room temperature, were performed using SRIM- $2013^{33}$ to show the D ions' influence on the cathode materials. The projected range (average penetration depth) and stopping energy (energy loss per $1 \mathrm{~nm}$ of the material surface) of $\mathrm{D}^{+}$inside Ti and SS surfaces are calculated and depicted as a function of $\mathrm{D}^{+}$energy in Fig. 8. The penetration depth of $\mathrm{D}^{+}$inside the SS $\left(8.03 \mathrm{~g} \mathrm{~cm}^{-3}\right)$ surface is less than that inside the Ti $\left(4.43 \mathrm{~g} \mathrm{~cm}^{-3}\right)$ surface, which can be explained by the material density effect. Ions

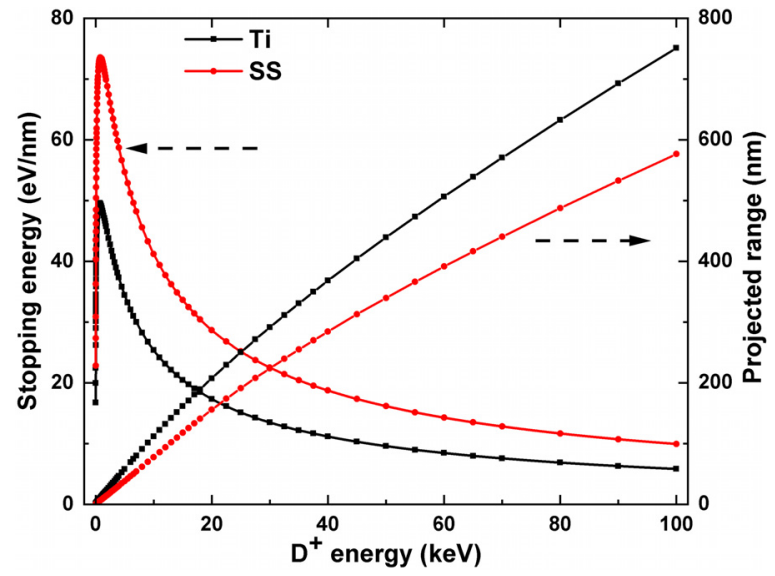

FIG. 8. Stopping energy and the projected range of $\mathrm{D}^{+}$ions with different energies in Ti and SS calculated by SRIM-2013. ${ }^{29}$ penetrate light material more deeply than dense material due to the heavy ions struggling into the material layers. Figure 8 shows clearly that the stopping energy of $\mathrm{D}^{+}$on the SS surface is drastically higher than on the Ti surface and that $\mathrm{D}^{+}$, with an energy $<1 \mathrm{keV}$, loses most of its energy within several $\mathrm{nm}$ from the cathode surface. When ions are hitting the cathode surface, the ions' kinetic energy is converted to thermal energy, generating heat on the surface. Therefore, the expected heating of the SS surface is higher than that of the Ti surface for the same given input power. The cathode temperature is continuously measured during the experiment, revealing that the SS surface temperature is higher than the Ti one for the same input power (see Table II). The difference in the specific electrical resistance between SS $(55 \mu \mathrm{Ohm} \mathrm{cm})$ and $\mathrm{Ti}(72 \mu \mathrm{Ohm} \mathrm{cm})$ cannot explain this large temperature difference, while the contribution of the deposited heat from $\mathrm{D}$ atoms on the grid surface may do.

Concurrently, the electron emission rate from the cathode surface increases and contributes to the atomic and molecular processes shown in Table I, affecting the NPR in the IEC space. Due to the low melting temperatures of $\mathrm{Ti} \sim 1600^{\circ} \mathrm{C}$ and $\mathrm{SS} \sim 1375^{\circ} \mathrm{C}$, it is difficult to use higher input powers to generate more neutrons, taking into consideration the additional heat generated from the ion circulation. For a given applied voltage and grid current, a cathode at higher temperatures may release adsorbed atoms near its surface faster than a cathode at lower temperatures. ${ }^{34}$ The projected range of $\mathrm{D}^{+}$in the Ti surface is higher than in the SS surface (Fig. 8), which increases the possibility of ions accumulating more deeply in the Ti grid than in the SS grid. From the above argument, the penetration depth of $\mathrm{D}^{+}$inside the $\mathrm{Ti}$ surface is considerably longer, the stopping energy is smaller, and the surface temperature is lower than in the SS surface. Therefore, the possibility of the Ti surface to lose adsorbed ions is minimal compared with the SS surface, leading to more surface fusion reactions and a higher neutron yield. The cathode material and temperature influence the $\mathrm{D}$ atoms' adsorption and desorption rates from the cathode surface, leading to a change in the surface fusion contribution in the total NPR. Therefore, a 2D heat diffusion simulation is needed to predict the effect of the grids materials' emission properties on the adsorbed and desorbed $\mathrm{D}$ atoms at the cathode surface. Moreover, considering all types of molecules inside the IEC space, $\mathrm{D}, \mathrm{D}_{2}, \mathrm{D}^{+}, \mathrm{D}^{+}{ }_{2}$, and $\mathrm{D}^{+}{ }_{3}$ are critical to identifying the IEC's predominant reaction. Obviously, the cathodes under investigation have different thermal properties, significantly affecting the cathode materials' influence on the fusion reactions.

From the present experimental configuration and results, it is hard to gauge the Moly rod's contribution, which was used to suspend the grid in the chamber center [Fig. 1(b)], to the total NPR. The rod appeared glowing during the entire experiment with the Ti and SS grids [Fig. 1(c)], which could be interpreted by the input power or

TABLE II. Cathode temperatures at different powers.

\begin{tabular}{lll}
\hline \hline Input power/cathode & $\mathrm{SS}\left({ }^{\circ} \mathrm{C}\right)$ & $\mathrm{Ti}\left({ }^{\circ} \mathrm{C}\right)$ \\
\hline $2.4 \mathrm{~kW}(30 \mathrm{kV}, 80 \mathrm{~mA})$ & $860 \pm 3$ & $810 \pm 2$ \\
$3.2 \mathrm{~kW}(40 \mathrm{kV}, 80 \mathrm{~mA})$ & $901 \pm 2$ & $852 \pm 4$ \\
$4.0 \mathrm{~kW}(50 \mathrm{kV}, 80 \mathrm{~mA})$ & $932 \pm 4$ & $884 \pm 3$ \\
$4.8 \mathrm{~kW}(60 \mathrm{kV}, 80 \mathrm{~mA})$ & $956 \pm 4$ & $920 \pm 4$ \\
\hline \hline
\end{tabular}


heating from the impacts of circulating ions. It is worth mentioning that the ability of the Moly to adsorb D is larger than in SS and smaller than in Ti. A future experiment is planned using a rod made from the same cathode material to avoid the possibility of rod contribution on the NPR.

When the applied voltage on the cathode rises, $\mathrm{D}^{+}$energy increases. Hence, the DD fusion cross section is expected to improve; simultaneously, the fusion rate between energetic ions with ions or molecules embedded significantly deeply in the cathode surface also increases; this explains the rise in the NPR/I-cathode ratio when the voltage increases, as seen in Fig. 7. The simulation results show that the ratio of ion penetration depths between $\mathrm{Ti}$ and SS, for $\mathrm{D}$ ions with $1 \sim 100 \mathrm{keV}$ energy, is comparable to the corresponding ratios in NPR/I-cathode ranges from 1.66 to 1.29 , with 1.49 on average. The above discussion on $\mathrm{D}^{+}$inside cathode materials may also be valid for neutrals or other molecules generated via charge exchange in the IEC space. One can conclude that the Ti cathode's affinity to retain D and neutrals on its surface is much larger than that of the SS cathode. As a result, the possibility of fusion reactions that generate neutrons on the cathode surface is higher for Ti than for SS. The above argument is supported by the experimental and simulation results, as seen in Figs. 7 and 8 . It is difficult, however, to precisely identify the influences of the different fusion reactions on the total NPR.

The Ti and SS cathodes in buckyball-shaped grids are worth using as cathode materials for an IEC device employed in neutron applications $<5 \times 10^{7} \mathrm{n} / \mathrm{s}$. However, applying higher input power than $6 \mathrm{~kW}$ to generate more neutrons is not recommended for Ti and SS grids. The higher input power leads to higher cathode temperature, which may cause melting of the grid. The Ti grid performs better than the SS grid due to the surface fusion reaction and the D adsorption effect. More investigation of NPR factors is needed to improve IEC fusion performance and thus increases its associated applications. Additional studies are urgently required to understand the gas target's dependency and surface target on cathode properties and transparency. 2D heat diffusion simulation is essential to evaluate the cathode thermal property effect on the NPR. Increasing cathode transparency enhances beam-background gas fusion while decreasing the beamsurface fusion and vice versa; such as quantifying this trade-off is still an open issue and optimization of the cathode transparency is needed. Directly comparing a conventional disk and a 3D printed grid of the same material and radius, with different surface areas, may help to elucidate this issue. An additional experiment is planned to optimize cathode transparency and shape to maximize the IEC neutron yield from 3D printed cathodes.

\section{SUMMARY}

Two buckyball-shaped cathodes fabricated from Ti and SS were investigated for the first time in a spherical IEC chamber. Cathode evaluation was performed by examining P-V curves, the NPR dependency on grid current, and the applied voltage. Additionally, a simple diffusion simulation was performed to determine the influence of the cathode material on the projected range and deposited energy of the $\mathrm{D}^{+}$ions inside the cathode surface. The achieved NPRs at a voltage of $70 \mathrm{kV}$ and a grid current of $80 \mathrm{~mA}$ from the IEC with Ti and SS cathodes, so far, were $2.32 \times 10^{7}$ and $1.41 \times 10^{7} \mathrm{n} / \mathrm{s}$, respectively. The normalized NPR from the IEC using the Ti cathode is 1.36-1.64 times higher than those for the SS cathode at an applied voltage of $20-70 \mathrm{kV}$.
These results were explained based on the cathode material properties and the beam-surface fusion reaction on the cathode surface. The results concluded that the density of ions and neutrals adsorbed and/ or embedded in $\mathrm{Ti}$ was much larger than that for SS, giving more fusion reaction on the cathode surface and hence the NPR. This study suggests the need to investigate further the factors affecting the NPR generated by the IEC fusion device to improve system performance. Determining the optimum cathode transparency, which maximizes beam-background fusion and beam surface fusion, is planned for future work, using 3D buckyball-shaped grids with varying transparency.

\section{ACKNOWLEDGMENTS}

The authors would like to thank J. Yagi, S. Okino, and Y. Ogino from the Institute of Advanced Energy, Kyoto University, for helping to conduct the work. This work was partially supported by a Grant-in-Aid for young scientists (20K14442) from the Japan Society for the Promotion of Science (JSPS).

\section{DATA AVAILABILITY}

The data that support the findings of this study are available from the corresponding author upon reasonable request.

\section{REFERENCES}

${ }^{1}$ W. C. Elmore, J. L. Tuck, and K. M. Watson, Phys. Fluids 2, 239 (1959).

${ }^{2}$ P. T. Farnsworth, U.S. patent 2,358,402 (June 1966).

${ }^{3}$ R. L. Hirsch, J. Appl. Phys. 38, 4522 (1967).

${ }^{4}$ K. Noborio, T. Sakai, and Y. Yamamoto, in Proceedings-Symposium Fusion Engineering (2003), pp. 328-331.

${ }^{5}$ K. Yamauchi, S. Ohura, M. Watanabe, A. Okino, T. Kohno, E. Hotta, and M. Yuura, Performance of Neutron/Proton Source Based on Ion-Source-Assisted Cylindrical Radially Convergent Beam Fusion (The Institute of Electrical Engineers of Japan, 2006), pp. 1177-1182.

${ }^{6}$ K. Noborio, Y. Yamamoto, and S. Konishi, Fusion Sci. Technol. 52, 1105 (2007).

${ }^{7}$ Y. Takahashi, T. Misawa, K. Masuda, K. Yoshikawa, T. Takamatsu, K. Yamauchi, T. Yagi, C. Ho Pyeon, and S. Shiroya, Appl. Radiat. Isot. 68, 2327 (2010).

${ }^{8}$ J. Hedditch, R. Bowden-Reid, and J. Khachan, Phys. Plasmas 22, 102705 (2015).

${ }^{9}$ M. K. Michalak, A. N. Fancher, G. L. Kulcinski, and J. F. Santarius, Fusion Sci. Technol. 72, 449 (2017).

${ }^{10}$ K. Masuda, R. Kashima, and M. A. Bakr, Fusion Sci. Technol. 75, 608 (2019).

${ }^{11}$ M. Bakr, K. Masuda, and M. Yoshida, Fusion Sci. Technol. 75, 479 (2019).

${ }^{12}$ S. Mukai, K. Ogino, Y. Yagi, and J. Konishi, IEEE Trans. Plasma Sci. 48(6), 1831 (2020).

${ }^{13}$ N. Ranson, V. Pigeon, N. Claire, and J. Khachan, Phys. Plasmas 27, 103501 (2020).

${ }^{14}$ Y. Takahashi, T. Misawa, C. H. Pyeon, S. Shiroya, and K. Yoshikawa, Appl. Radiat. Isot. 69, 1027 (2011).

${ }^{15}$ Y. Nakai, K. Noborio, Y. Takeuchi, R. Kasada, Y. Yamamoto, and S. Konishi, Fusion Sci. Technol. 64, 379 (2013).

${ }^{16}$ G. H. Miley, H. Momota, H. Leon, B. Ulmen, G. Amadio, A. Khan, G. Chen, W. Matisiak, A. Azeem, and P. Keutelian, J. Eng. Gas Turbines Power 133, 124502 (2011).

${ }^{17}$ R. Bowden-Reid, J. Khachan, J. P. Wulfkühler, and M. Tajmar, Phys. Plasmas 25, 112702 (2018).

${ }^{18}$ D. C. Donovan, D. R. Boris, G. L. Kulcinski, and J. F. Santarius, Fusion Sci. Technol. 56, 507 (2009).

${ }^{19}$ K. Yoshikawa, K. Masuda, T. Takamatsu, S. Shiroya, T. Misawa, E. Hotta, M. Ohnishi, K. Yamauchi, H. Osawa, and Y. Takahashi, Nucl. Instrum. Methods Phys. Res., Sect. B 261, 299 (2007). 
${ }^{20}$ B. J. Egle, J. F. Santarius, and G. L. Kulcinski, Fusion Sci. Technol. 52, 1110 (2007).

${ }^{21}$ J. Rasmussen, T. Jensen, S. B. Korsholm, N. E. Kihm, F. K. Ohms, M. Gockenbach, B. S. Schmidt, and E. Goss, Phys. Plasmas 27, 083515 (2020).

${ }^{22}$ M. Bakr, K. Masuda, and M. Yoshida, AIP Conf. Proc. 2160, 030004 (2019).

${ }^{23} \mathrm{~J}$.-P. Wulfkuehler and M. Tajmar, in 52nd AIAA/SAE/ASEE Joint Propulsion Conference (American Institute of Aeronautics and Astronautics, Reston, Virginia, 2016).

${ }^{24}$ J. Kipritidis, J. Khachan, M. Fitzgerald, and O. Shrier, Phys. Rev. E 77, 066405 (2008).

${ }^{25}$ J. Khachan and S. Collis, Phys. Plasmas 8, 1299 (2001).

${ }^{26}$ J. Kipritidis and J. Khachan, Phys. Rev. E 79, 026403 (2009).

${ }^{27}$ D. R. Boris and G. A. Emmert, Phys. Plasmas 15, 083502 (2008).
${ }^{28}$ R. L. Hirsch, Phys. Fluids 11, 2486 (1968).

${ }^{29}$ P. W. Tamm and L. D. Schmidt, J. Chem. Phys. 55, 4253 (1971).

${ }^{30}$ K. Christmann, O. Schober, G. Ertl, and M. Neumann, J. Chem. Phys. 60, 4528 (1974).

${ }^{31}$ A. Y. Didyk, R. Wiśniewski, K. Kitowski, V. Kulikauskas, T. Wilczynska, A. Hofman, A. A. Shiryaev, and Y. V. Zubavichus, Phys. Part. Nucl. Lett. 9, 86 (2012).

${ }^{32}$ W. Espe, Electronics (2016), see https://vacaero.com/information-resources/ vac-aero-training/1166-getter-materials.html.

33J. P. Biersack and L. G. Haggmark, Nucl. Instrum. Methods 174, 257 (1980).

${ }^{34}$ Q. Zhou, A. Togari, M. Nakata, M. Zhao, F. Sun, M. Yajima, M. Tokitani, S. Masuzaki, N. Yoshida, M. Hara, Y. Hatano, and Y. Oya, Int. J. Hydrogen Energy 45, 9959 (2020). 